\title{
THIELAVIOPSIS ROT OF BANANAS
}

Green banana hands of the Niño (Lady-finger, Sour criere) variety, showing evidence of a black rot of the stalk cushion and the finger-stalk, were placed in moist chambers for observation. The infection progressed rapidly and in a few days the fruit ripened prematurely and turned black with the pulp becoming brown and mushy.

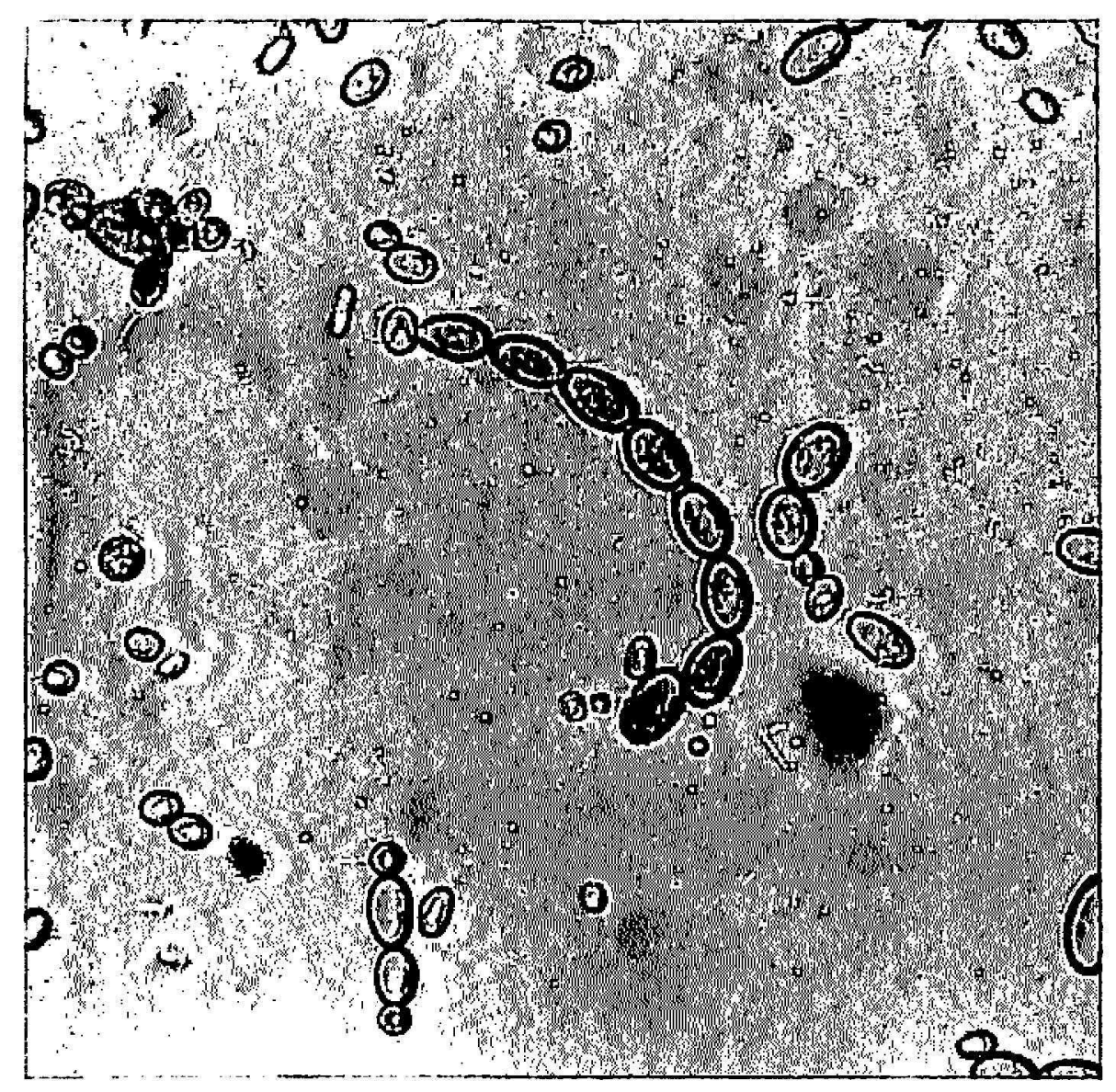

Fig. 1.-Catenulated, dark macroconidia-also known as chlamydospores-and a few, hyaline endoconidia of Ceralocystis paradoxa Bakshi.

The bunches from which the affected material came were harvested in rainy weather unprotected from soil contamination.

The ubiquitous fungus, Thielaviopsis paradoxa (De Seynes) Hohn, was isolated from the cushions, the finger-stalks and the mushy pulp of the banana fruits. The pathogenicity of this organism was corroborated when healthy banana hands were inoculated through wounds on the cushions. This is the first instance this fungus has been found 10 affect bananas in Puerto Rico. T. pararloxa also causes serious damage to sugarcane and pineapples in P'uerto Rico. 
The organism can live in the soil in host-plant residues. It produces two kinds of spores under field conditions: 1, Endoconidia of a delicate nature and liable to loss of viability if the environment is not favorable for immediate infection; 2, chlamydospores of a very resistant nature, consequently capable of withstanding adverse weather conditions (fig. 1). A third type of spore, the ascospore, is produced by the fungus in its perfect, ascomycetous stage, i.e., Ceratocystis paradoxa Bakshi (fig. 2).

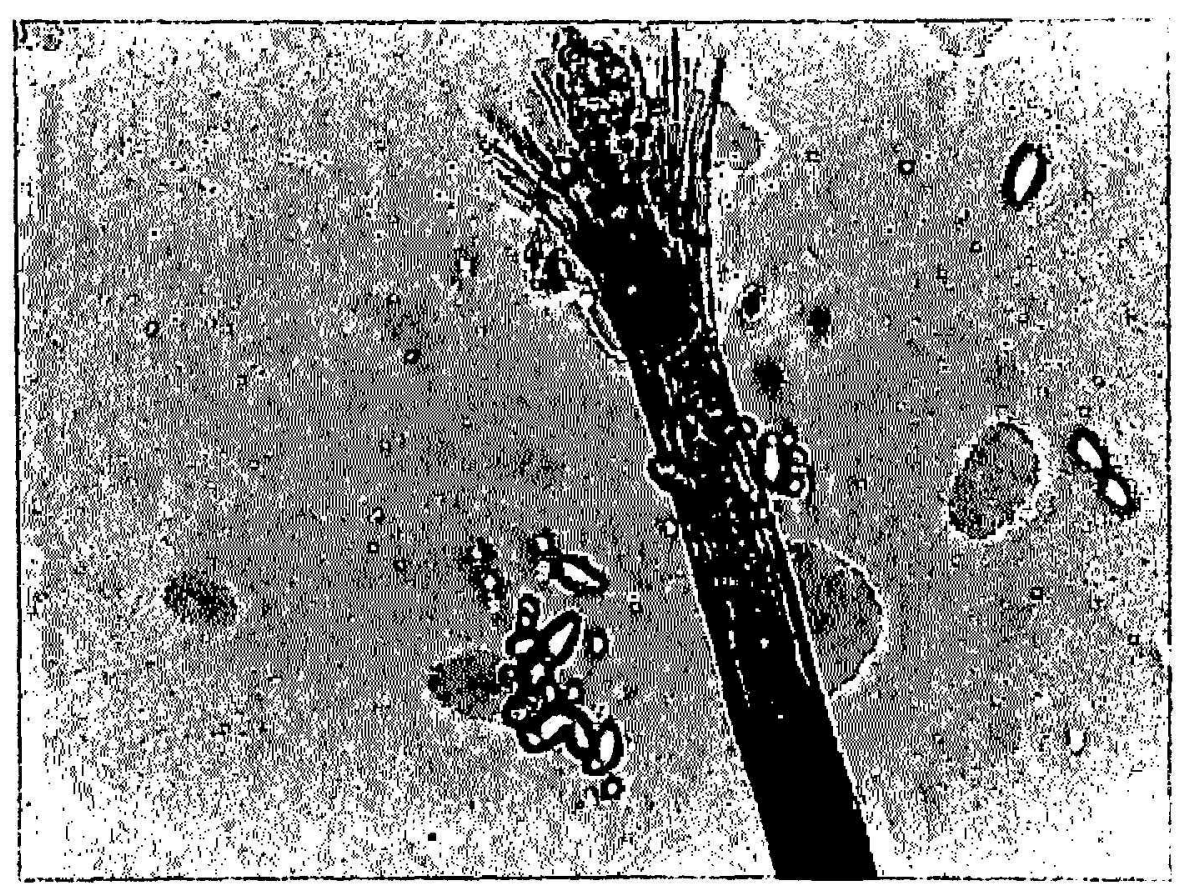

Fig. 2.-Hyaline ascospores extruded through the brush-like tip of the neck of a perithecium of Ceratocystis paradoxa Bakshi.

The fungus is a wound parasite found in the soil in its chlamydosporic stage. It readily causes infections if these spores come in contact with wounded tissues of susceptible plants.

Infections of bananas can be avoided if the bunches do not get soiled during harvest, if the hands are cut with clean knives, and if the hands severed from the bunches in packing-center surroundings are kept free from banana, pineapple and sugarcane waste products which serve as media for the growth and reproduction of $T$. paradoxa.

Luis A. Alvarez-Garcia

Departmenl of Phylopalhology and Botany 